\title{
Review
}

\section{Conversion motor paralysis disorder: overview and rehabilitation model}

\author{
RJ Heruti ${ }^{1,4}$, A Levy $^{2,4}$, A Adunski ${ }^{3,4}$ and A Ohry*,1,4 \\ ${ }^{1}$ Rehabilitation Ward, Reuth Medical Center, Tel-Aviv, Israel; ${ }^{2}$ Shalvata Psychiatric Hospital, Hod-Hasharon, Israel; \\ ${ }^{3}$ Geriatric Department, Sheba Medical Center, Tel-Hashomer, Israel; ${ }^{4}$ Sackler Faculty of Medicine, Tel-Aviv \\ University, Israel
}

It is important to consider a differential diagnosis between paralysis on an organic basis and paralysis and disability due to psychological mechanisms in people with physical impairment secondary to trauma, without evidence of organic etiology. We review the most dramatic type of conversion disorder (CD)- 'Conversion Motor Paralysis'. Recent important medical literature concerning the accepted treatment and rehabilitation management will be reviewed and discussed. The inter-disciplinary in-patient team management approach in a rehabilitation setting offers the benefits of a comprehensive assessment and treatment. The diagnosis is temporary and conditional, since there may be a long delay until the appearance of organic findings. A complete medical assessment is essential in order to rule out any possibility of an organic etiology. In as many as $25 \%$ to $50 \%$ of patients diagnosed as conversion, an organic medical diagnosis was found.

Spinal Cord (2002) 40, 327-334. doi:10.1038/sj.sc.3101308

Keywords: conversion disorder; motor paralysis; rehabilitation model; interdisciplinary team approach; overview

\section{Introduction}

Patients with physical impairment secondary to trauma, without evidence of organic etiology are seldom referred to rehabilitation centers. On those occasions it is important to consider a differential diagnosis between paralysis on an organic basis and paralysis and disability due to psychological mechanisms. Management may be affected at several levels:

1. Diagnostic (differential diagnosis, ruling out organic causes).

2. Treatment (psychiatric, psychological, rehabilitation, long-term follow-up).

3. Administrative (determination of paralysis benefit due to a conversion disorder may, or may not, be legally similar to other forms of paralysis, depending upon the payer, or health care system).

4. Social and occupational issues.

Accurate diagnosis is imperative as erroneous diagnostic labeling may expose these patients to unnecessary treatments and the potential for serious side effects. These in turn may have a long-term detrimental impact on medical management. Even without an organic basis for their signs and symptoms

*Correspondence: A Ohry, PO Box 2342, Savyon 56530, Israel these patients often require comprehensive assessment, treatment and rehabilitation.

Rehabilitation is an expensive and time-consuming process involving a highly skilled multi-disciplined team as well as a detailed and complete diagnostic follow-up. ${ }^{1-4}$ The inter-disciplinary team approach ${ }^{5}$ in a rehabilitation center offers distinct advantages in the treatment of conversion disorders (CD). CD is similar to organic disabilities in that it affects the occupational and social aspects of the patient's life. In addition to the obvious benefits of a comprehensive approach, the stigma of psychiatric hospitalization is avoided. Initially, in many cases neither the patient nor the treating staff are aware of the conversive etiology. Patients suspected of malingering or having secondary gain from their disability must be differentiated from those with CD.

We review recent as well as historically important medical literature concerning patients sustaining the most dramatic type of CD - motor symptoms, weakness or paralysis for instance - 'Conversion Motor Paralysis'. These cases are relatively rare and there is no precise epidemiological mapping of the prevalence and incidence. ${ }^{2,4}$ The differential diagnosis, pathophysiology, potential psychiatric co-morbidities, accompanying disabilities (mental and others), management and rehabilitation aspects are reviewed and discussed. 


\section{Overview}

\section{History of hysteria}

Hysteria was first documented 4000 years ago by the Egyptians, who believed the symptoms originated from the uterus, hence the name (hysterus). ${ }^{2}$ 'Hysterical' conditions included combinations of seizures, paralysis, and anesthesia. During the 17th and 18th centuries a variety of disorders, such as: hypochondriasis, hysteria, dyspepsia, 'gas and spleen disease' (Vapours) were included in the general term 'Nervous Disorder', a term created by Briefe in 1603 , that was subsequently replaced with the vague term 'Nervous Temperament'. nerve pathologies commenced in 1843, the year $\mathrm{Du}$ Bois Reymond demonstrated electrical conduction in nerves. No electrical disturbances were found in the 'Nervous Temperament', leading to the hypothesis of psychogenic origin. ${ }^{7}$ The term 'Hysterical Conversion' was created about 100 years prior to Freud's birth, in an attempt to justify the existence of hysteria as a diagnosis. The French neurologists, Babinski and Charcot were among the first to publish articles concerning hysteria in the modern literature. Lhermitte wrote 'hysteria is the mother of deceit and trickery'. Babinski removed from hysteria some components, such as; secondary physical findings, malingering, self-injuries and pathological lies. $\mathrm{He}$ defined hysteria as a disease with a psychological etiology, and no clear physiological or morphologic evidence, and characterized the hysterics as hypersuggestible and easy to hypnotize. ${ }^{6,7}$ Paul Briquet was the first to make an association between $C D$ and central nervous system disorders during the 19th century. He claimed $\mathrm{CD}$ was due to stress and environmental situations, affecting 'affective' areas in the brain of person with pre-morbid hypersensitivity. ${ }^{8}$ His follower, Charcot, hypothesized that these patients were suffering from a global disorder of the brain, exposing them to the development of $\mathrm{CD}$. $\mathrm{He}$ developed the primary description of hysteria and contributed to the understanding, diagnosis and management of this disorder. Lately, his work has regained recognition, when the component of his theory concerning the pathophysiology of trauma was introduced into the modern theories regarding post-traumatic stress disorder (PTSD) and Somatoform Disorder. ${ }^{9}$

Freud, a student of Charcot, defined 'La Grande Hysterie', that overlapped the definition of motor paralysis. Freud's concept of conversion originated from an integration of medical thoughts and knowledge in this area during the 19th century, which led him to create the term 'Conversion Neurosis'. According to his traumatic model of hysteria, published in 1899, hysterical symptoms stem from sexual trauma, that activates an old traumatic event (Nachtriglichkeit). Freud argued that through analysis the childhood trauma is restored and the neurotic symptoms released via a transfer mechanism. ${ }^{10}$
History itself has provided evidence of women with extra-ordinary achievements, all of them with hysteria, such as Theresa of Avila and Florence Nightingale. ${ }^{7}$ There are reports of Mass Hysteria, mostly during wars or crisis. ${ }^{11}$ Of those with less favorable achievements, Adolf Hitler can be mentioned, as suffering from hysterical blindness.

\section{Definitions}

There are a variety of psychological disorders, which may result in physical disability without any organic basis. Confusion has arisen because of the multitude of terms and definitions, as well as overlap between historical and current terminology. While in the past the term hysteria was used in multiple contexts, the following is the current accepted terminology: ${ }^{4}$

(a) Somatization Disorder - Classic historical hysteria (Briquet's syndrome)

(b) Hysterial Neurosis - including:

(1) Conversion Disorder (CD)

(2) Dissociative Disorder

(c) Anxiety Hysteria - includes Freud's definition of phobia

(d) Hysterical Personality - a term that was substituted by the term Histrionic Personality.

Most terms included in the above terminology are associated with the present term 'Somatoform Disorder'. The definitions used come from DSM-IV concerning a group of disorders, characterized by somatic symptoms not adequately explained by a disease, side effects of medication or due to any other psychological mechanism (ie Panic Disorder). The symptoms cause significant distress, affecting different aspects of life (functional, social and occupational). Unlike Factitious Disorder or Malingering, the somatic symptoms are not deliberate (the patient has no voluntary control over their production). Similar psychological symptoms might accompany other general diseases, but neither disease nor medical condition explain the somatic symptoms found in patients suffering from Somatoform Disorder. This group includes several conditions, each sharing the absence of an organic disease as an etiology for the symptoms. ${ }^{4}$

The term Conversion Disorder (Hysterical Neurosis - Conversion type) is listed in the DSM-IV ${ }^{4}$ under Somatoformic Disorder group (code 300.11), and is described as a psychological disorder, characterized by somatic symptoms with no physiological abnormalities, but with an underlying psychological basis. Most of the conversion symptoms (CS) are neurological and usually relate to the loco-motor system. The motor symptoms include convulsions, paralysis, weakness, dyskinesia; sensory symptoms include paraesthesia or anesthesia, blindness or speech disorders. These symptoms normally suggest the existence of a neurological disorder or other similar medical condition. 
The term Conversion State is used when there is an unconscious simulation of an organic disorder. When it appears together with an organic disease, it is regarded as functional overlay.

\section{Pathophysiology}

Several theories were constructed in order to explain the etiology of $\mathrm{CD} .^{2}$ By constructing the theory regarding the existence and action of the unconscious, and based on his experience with conversion patients, Freud has provided the understanding of the psychodynamic background and etiology of CD. Suppression is the major defense mechanism involved in conversion, as noted by the close relation between conversion conditions and traumatic events in the patient's past. ${ }^{12}$ According to Freud, an impulse or a wish, that cannot be fulfilled due to negative connotations (such as, fear, shame, guilt or anger) is converted into physical expression, so that the CS actually reflects a symbolic solution to the same unconscious psychological conflict. Freud accentuated the symbolic relation existing between the type of the CS and the conflict. ${ }^{13,14}$

Freud defines primary and secondary gains a patient receives: Primary gain - anxiety produced by an internal unconscious defensive mechanism is converted into symbolic physical symptoms, while the conflict remains limited within the unconscious, thus resulting in reduction of the anxiety level.

Secondary gain - achieved by avoiding certain obligations or by getting support from being a patient (ie, avoiding work or responsibility, getting care, attention etc).

\section{Epidemiology}

The frequency of $\mathrm{CD}$ varies among different reports from $11 / 100000$ to $300 / 100000$ according to the population type. ${ }^{2,4}$ The annual incidence in Monroe county, New-York was 22/100 000 cases, compared to $11 / 100000$ in Iceland during $1960-1969 .{ }^{15}$ CD accounts for up to $1 \%-3 \%$ of patients in psychiatric outpatients clinics. ${ }^{16}$ Hafeiz described a frequency of $10 \%$ found in a sample patients taken 3 years, ${ }^{17}$ but there are reports of up to $20 \%$ among patients referred to various evaluation procedures. ${ }^{18}$

There are divided opinions on the male-female frequency of $\mathrm{CD}$. It would appear to be more frequent in females, with reports varying from $2: 1$ to $15: 1,{ }^{17,18}$ although other reports state that there is no difference. ${ }^{19}$

$\mathrm{CD}$ was described as more frequent in rural areas, among low socio-economic status, and among subjects with less medical and psychological knowledge. ${ }^{4}$ High frequency of $\mathrm{CD}$ was found among Apalash men, ${ }^{20}$ and females in Sudan. ${ }^{17}$ Several modes of CD, such as a fall accompanied by loss of consciousness, are related to cultures, where CS expresses anxiety. Thus, it is important to evaluate the relation between the CS and cultural context.
According to DSM-IV the risk factors include previous physical disability, exposure to other disabled subjects and extreme psycho-social anxiety. ${ }^{4}$ There is limited information concerning a higher frequency of $\mathrm{CD}$ among relatives of subjects with $\mathrm{CD}$. A higher frequency in monozygotic twins, but not among dizygotic $^{4}$ has, however, been reported. Ljundberg was the first to demonstrate higher rate of CS among first-class relatives of conversion subjects $(12-14$ times in females and 4-6 in males), arguing for an hereditary tendency, in addition to environmental causes. ${ }^{21}$

Usually CD appears in adolescence or young adulthood. Presentation before the age of 10 or after 35 is rare, though rarely cases have been reported in the ninth decade. ${ }^{4} \mathrm{CD}$ in children below the age of 10 is usually limited to walking impairments ${ }^{22}$ or convulsions.

\section{Clinical presentation}

Subjects suffering from CD might behave in a way known as 'la belle indifference', a situation in which the patient appears detached from the physical symptoms, that otherwise would have caused him great anxiety. Other presentations are the dramatic of histrionic. The intensity of the disability is usually to a level that affects activities of daily living. CS is often aggravated by anxiety and tension states, such as the death of a relative or a war situation.

During the course of the disease the subject tends to dependant behavior or adopts 'the patient role'. Accompanying psychological symptoms are abundant and include dissociative disorder, depression, and personality disorders (especially borderline anti-social and dependant). ${ }^{4}$ The symptoms are divided according to the dominant clinical presentation.

In this review $\mathrm{CD}$ with motor symptoms is the major focus. Motor symptoms might include equilibrium or coordination impairment, weakness or paralysis, vocal disorders (hoarseness to aphonia), dysphagia or a choking sensation in the throat, and urinary incontinence. ${ }^{4}$ CS does not usually follow known anatomical or physiological routes. It is characterized by inconsistency and instability of the presence and severity of the signs and symptoms. Paralyzed limbs might move 'accidentally' while performing activity or when attention is shifted. ${ }^{23,24}$

Conversion Gait Disorder. This disorder consists of uni- or bilateral lower limb weakness, with possible accompanying ataxia and/or tremor. The gait does not usually resemble any pathological gait of known neurological origin, and is easily diagnosed by a clinician experienced in CD. According to Keane, who described 60 patients with conversion gait disorder, dramatic recovery is a major sign directing towards a conversion origin. Dystonia and chorea are the most common conversion symptoms among children, and are more difficult to diagnose. ${ }^{23}$ Often diagnosis is delayed, and may involve many unnecessary diagnostic tests. ${ }^{22}$ 
Limb Paralysis. Several reports concerning conversion limb paralysis have been found in the literature. Cardenas et al describe upper limb paralysis among chronic pain patients. ${ }^{25}$ Withrington reports five women, suffering limb weakness without accompanying muscle atrophy. ${ }^{26}$

Other forms of conversion motor disorders described in addition to those mentioned above, include vocal disorders (hoarseness, dysphasia, aphonia, etc), ${ }^{27}$ bronchospasm (characterized by recurrent stridor attacks in youth, accompanied by panic reaction), ${ }^{28}$ and motor visual disorders (very rare, and expressed as blepharospasm). ${ }^{29}$

Other sub-groups of $\mathrm{CD}$ include sensory loss or signs such as anesthesia, paresthesia, visual impairment (mainly amblyopia), ${ }^{29}$ blindness, deafness, hallucinations, ${ }^{30-31}$ sleep disorders, ${ }^{32}$ etc. The symptoms can 'travel' between systems, for example, vocal disorder 'recovers' and is replaced by pathological gait.

\section{Diagnosis}

The diagnosis is determined after ruling out organic components or other psychogenic diagnosis. ${ }^{1}$ The diagnostic process consists of precise medical history, thorough physical examination and the use of appropriate diagnostic tools.

The diagnostic criteria according to DSM-IV to determine $\mathrm{CD}$ are: ${ }^{4}$

- One or more symptoms or deficits affecting voluntary motor or sensory function that suggest a neurological or other general medical condition.

- Psychological factors judged to be associated with symptoms or deficit because the initiation or exacerbation of the symptoms or deficit is preceded by conflicts or other stresses.

- The symptom or deficit is not intentionally produced or feigned (as in Factitious Disorder or Malingering).

- The symptoms or deficit cannot, after appropriate investigation, be fully explained by a general medical condition, or by the direct affects of a substance, or as a culturally sanctioned behavior or experience.

- The symptom or deficit causes clinically significant distress or impairment in social, occupational, or other important areas of functioning or warrants medical evaluation.

- The symptom or deficit is not limited to pain or sexual dysfunction, does not occur exclusively during the course of Somatization Disorder, and is not better accounted for by another mental disorder.

The usual symptoms are motor and/or sensory losses ('pseudo-neurological' symptoms), but convulsive and mixed presentation also exist. It should, however be remembered that DSM-IV is updated regularly, and overlaps may occur between several disorders.

The diagnosis is always temporary and conditional, due to the time factor involved until the appearance of organic evidence (as in systemic disease). Of importance is the fact that between 25 and $50 \%$ of patients diagnosed as conversion, will subsequently be diagnosed with an organic medical condition. ${ }^{33,34}$ This percentage is lower in recent publications, most probably because of increased awareness to the disorder, concurrent with advances in diagnostic techniques. The possibility that a present symptom is conversion increases, if the patient has a history of unexplained physical complaints (especially conversion) or dissociative symptoms, and if he/she meets the criteria for Somatization Disorder. ${ }^{35}$ The less the patient's medical knowledge, the more he/she appears with inexplicable and irregular symptoms. In contrast, symptoms of educated patients are very similar to genuine ones. ${ }^{3}$

Despite all the above, the diagnosis is frequently clinical, based on history and physical examination. ${ }^{3,24,36}$

History - Supporting indicators in the patient's history include: previous functional disorders, role models among family member or friends, secondary gain, professional relation to medicine (in Baker's series $5 / 26$ women were nurses), ${ }^{24}$ previous psychiatric background, and self-discharge from hospital. The onset is usually acute and sudden, though there may be a gradual form. ${ }^{20}$

Other details in the patient's history compliant to a diagnosis of $\mathrm{CD}$ include mental retardation, concurrent psychiatric disease, physical or psychological trauma in childhood or close to the presentation of CS, and family history of physical disability. ${ }^{4}$

Physical examination - The signs leading towards a non-organic diagnosis include inconsistent findings, no adjustment between physical and functional findings, and inconsistency between the symptoms and anatomical or physiological systems. ${ }^{20}$ Objective signs, such as pathological deep tendon reflexes are rare. Signs seen more frequently are imitation of real symptoms from observation (eg, of convulsions of another patient). CS will rarely lead to physical changes or disability, so pressure sores, contractures or muscle atrophy are rare. Dysphagia will be similar for both solids and liquids. 2,20,37

In order to reach a correct diagnosis a thorough neurological examination is essential. Prominent suspicious symptoms include: jerky sharp movements, unexplained tremor, inconsistency in findings (eg, muscle strength of quadriceps in standing compared to sitting), bizarre gait pattern, slow motion movements, overflow of emotion during the exam (painful expression, tooth grinding, breath holding, etc). ${ }^{38}$ Other signs include normal muscle tone, normal reflexes, flexor plantar reflexes, bizarre movements, and simultaneous contraction of agonist and antagonist muscles (if the patient is asked to flex the elbow, 
contraction of the triceps, preventing flexion, is noticed. ${ }^{23,24}$ When the examiner attempts to change an abnormal limb position, a resistance in direct relation to the power exerted is felt. ${ }^{20,37}$ If a 'paralyzed' arm is raised above the subject's head and released, it will not fall directly on his head, but to the side.

The sensory examination might reveal changes not related to anatomical dermatomes, inconsistency in repeated examinations, misleading proprioception. Conversion anesthesia in a hand or foot will have a shape of a glove or sock, affecting all types of sensation, with no determinant level, and with a sharp border, and not according to dermatomal levels. ${ }^{20,37}$

The autonomic system is usually unaffected with full sphincter control and normal bowel movement. No spinal shock or other typical autonomic signs (eg, low pulse and blood pressure, dyspnea) are presented in conversion tetraplegia. ${ }^{37}$

Additional examinations - There are no pathological findings in laboratory tests, supporting CD. On the other hand, however, pathological findings will not necessarily rule out $\mathrm{CD}{ }^{37}$

Additional tests, ie, imaging (X-ray, CT, MRI) and electrophysiological studies (electroencephalography, sensory and motor evoked potentials, urodynamics) are usually normal, however, presence of findings rarely elucidate the clinical symptoms. When a subject is admitted with paraplegia, normal reflexes and full control of sphincters, a routine X-ray is sufficient, and the diagnosis is clinical. CT and MRI are unnecessary, and are performed just as additional supporting evidence for the clinical diagnosis. ${ }^{23,36}$

\section{Differential diagnosis}

According to Lazare the classic characteristics of CD, such as symbolization of the symptoms, secondary gains, histrionic personality, 'La Belle Indifference', are of no diagnostic power and are unnecessary. ${ }^{12}$ On the other hand, symptoms considered as conversion, such as sensory loss with no anatomical pattern, suggestibility, etc may accompany diseases of the nervous system. $^{34}$

Considering the differential diagnosis of $\mathrm{CD}$ there are two major groups: $:^{4,33}$

Organic background - A thorough evaluation in order to rule out organic etiology must be performed, before determining a diagnosis of psychogenic origin. Potential general medical conditions (eg, multiple sclerosis, myasthenia gravis, idiopathic dystonias) must be carefully excluded. ${ }^{1,4,34}$

Psychological background - Two main sub-groups can be noted in this group ie, voluntary and involuntary behavior. The decision whether a certain symptom is voluntary (factitious, malingering) or not, is difficult and is usually reached only after a thorough evaluation relating the context of the symptom presentation, especially in the presence of some primary gain. ${ }^{1}$ It is useful to crosscheck the patient's report with other sources (such as, friends, team members, etc). As mentioned above, signs that are limited to pain or sexual dysfunction are diagnosed as somatoform pain disorder or sexual dysfunction respectively, and not as CD. ${ }^{4}$

Voluntary behavior - In order to differentiate between factitious disorder and malingering it is important to assess the patient's awareness, the intention of the CS and their motivation. ${ }^{4}$

Factitious disorder is suspected when there is a partial combination of the following: dramatic and unfamiliar behavior, symptoms and behavior appear and are aggravated in the presence of a team member, pseudologia fantastica rebellious behavior (opposing procedures, arguing with staff, etc), great knowledge in medical terminology and routines, drug or alcohol abuse, evidence of many previous hospitalizations, small amount of visitors, and fluctuating clinical course, accompanied by aggravation of symptoms when primary evaluation is negative. Often it may be difficult to differentiate between factitious and conversion disorders. ${ }^{39}$

Factitious disorder is characterized by physical or mental symptoms produced voluntarily by the patient, while the unconscious motive is accepting the patient role. There is no evidence of external incentive (ie, compensation, avoiding legal responsibility). Proving a certain symptom is voluntarily counterfeit is made through direct evidence, whilst ruling out organic etiology. ${ }^{4}$ The symptoms most likely include forgery of complaints (abdominal pain, in the absence of pain), self-infliction (ie, injecting infectious substance in order to cause abscess), aggravating symptoms of presenting disease (false and exaggerated convulsions in an epileptic subject) or combination of the above. There are two forms: (a) Munchausen syndrome - chronic with physical signs. (b) Ganser syndrome - characterized by mental symptoms, often psychotic, that are not directed toward a familiar disorder. ${ }^{39}$ There is great variability in the nature of the symptoms, they do not respond to treatment, and are aggravated when the patient notices that he is being observed. The patient presents his suffering dramatically, but in an inconsistent manner and embedded with lies (Pseudologia Fantastica). Usually he has wide medical knowledge. When the primary evaluation is negative, new symptoms will appear. When the true nature of the illness is revealed (ie, direct evidence of self-infliction, identifying recurrent admissions etc), the patient will deny all proofs and will discharge himself hastily, against the physician's opinion, and will probably hospitalize himself in a different hospital. Often the course is chronic with an onset at young age. ${ }^{4,39}$

With malingering, the motive for producing the symptoms is conscious, and the purpose is prominent with clear secondary gain (eg, compensation, vacation from the army, release from the jail, etc) The patient has a clear external motive, in contrast to the factitious patient, who is motivated by the mental unconscious need to take on the patient role and 
receive treatment. Factitious reflects pathophysiology, whilst malingering reflects adjustment to certain condition (capture, jail).

Involuntary behaviour - The differential diagnosis of involuntary CS include depression, Panic Attack, ${ }^{40}$ PTSD,${ }^{41,42}$ Dissociative Disorder, ${ }^{4}$ and Borderline Personality. ${ }^{35}$

\section{Treatment and rehabilitation}

In the past, patients were referred to psychiatric departments, but this trend was changed, and due to the functional loss patients are referred to rehabilitation wards. ${ }^{43-45}$ The majority of papers on the topic are retrospective studies or case reports, authored by psychiatrists or specialists in physical medicine and rehabilitation. There are no long-term follow-up studies. The reference to treatment is minimal, contradicting and vague, offering autosuggestion, placebo, and hypnosis as the main treatments of choice. When the psychological intervention fails, inter-disciplinary rehabilitation treatment becomes even more of an imperative. $^{5}$ It is our impression that treatment should be directed towards the symptoms and as such the patients should be hospitalized in the correct units according to their physical symptoms, ie paraplegic or tetraplegic patients in a rehabilitation spinal unit, hemiplegic patients in a rehabilitation stroke unit etc and not placed in psychiatric units from the offset. Treatment in a rehabilitation system will also address the prevention of secondary disabilities. Those disabled due to conversion have many similarities to those with an organic basis for the disability, with regard to effects on physiology, social and occupational consequences.

Rehabilitation treatment should be introduced as early as possible. ${ }^{26}$ There is the need to rule out neurological, orthopedic and other potential medical etiologies. The patients must be screened and diagnosed, and to exclude those with suspected factitious disorder, malingering, or where there is secondary gain. Team members should be educated concerning the unconscious origin of the CS and the type of approach to be used in the treatment. ${ }^{43}$ The preferred setting is hospitalization in a rehabilitation ward in order to observe the patients in all activities. ${ }^{26,44-46}$ When both the medical etiologies and the conscious psychological etiologies are excluded, we are left with those patients disabled due to conversion disorders (impaired vision, balance deficits, difficulty in walking, limb paralysis, etc).

Three main treatment approaches should be considered:

The behavior modification approach ${ }^{2,47,48}$ The objectives underlying this approach are a reduction in the unwanted behaviors and a strengthening of the more desired behaviors. In order to achieve these objectives it is important to reward the more desired behaviors. ${ }^{47-49}$ Rather than punishing the unwanted behaviors they should, as much as possible, be ignored or at least not bring the desired focus of attention. A focus on the positive not the negative should be encouraged, and this may be achieved by the use of 'achievement charts' carefully screened obtainable goals, videotape feedback, in order to demonstrate the progress. ${ }^{26,50}$ It is preferable to treat the patient in a quiet place, away from the main treatment area, in order to avoid acquired behavior. ${ }^{51}$

Treatment using this approach begins even during the initial assessment when a diagnosis of $\mathrm{CD}$ is suspected due to the anomalies discovered on physical assessment. The diagnosis given should be vague rather than confrontational, ie in the case of hysterical paralysis 'spinal cord concussion', allowing the patient to undergo 'a speedy recovery'. ${ }^{48-49}$

Communication within the inter disciplinary team is essential and regular team meetings to document the progress of the patient should be held. Any differences of opinion between team members must be discussed only during these meetings. ${ }^{48} \mathrm{~A}$ case manager, possibly the psychologist, must direct and coordinate all treatments.

The psychotherapeutics approach The object of this approach is to resolve the conflict which has led to the clinical picture of CD. Psychotherapeutic treatment is given on an individual basis and its success will depend, to a large extent, on the cooperation of the patient. Drug therapy in the form of Thiopentone has proven itself to be an effective tool. ${ }^{52}$ Others report success with auto-suggestive treatments and hypnosis. ${ }^{53}$

The physical approach It is useful to combine the above approaches with a physical therapy approach. ${ }^{17,54-55}$ Exercises as per the 'vague diagnosis' may be prescribed together with functional electrical stimulation (FES) ${ }^{55}$ evoked potentials ${ }^{56}$ and biofeedback techniques. ${ }^{16}$ It is essential that regular objective measures be taken of the patient's functional abilities and these will have a great impact on the recovery outcome of the patient. Other objective measures in the form of balance testing or gait analysis are also important, since the results shown by the patient can be compared to the normative data available. As stated earlier the most successful approach to treatment would seem to be a combination of these three approaches.

\section{Prognosis}

Various reports on the prognosis of patients with $\mathrm{CD}$ are available. ${ }^{26,55}$ Some report hospitalization for weeks, and others months and yet others on spontaneous recovery within 2 weeks, without any treatment intervention. ${ }^{4,50,57}$ It should be stated, however, that the longer the time to recovery, the less complete will be the recovery. ${ }^{17}$ Reports have shown that between $15 \%$ and $75 \%$ of $\mathrm{CD}$ patients demonstrate organic signs within 5 years of diagnosis due to failure to recover or recurrence. ${ }^{17,20,50,51,53}$ 
Factors indicating favorable prognosis include sudden onset, presence of stressogenic factor during onset, short duration between diagnosis and onset of treatment, ${ }^{49}$ high level of intelligence, ${ }^{2}$ absence of definite psychiatric disorder, ${ }^{2,17}$ and aphonia and blindness as presenting CS. Poorer prognosis is related to severe disabilities with long duration, ${ }^{17}$ age above 40 years ${ }^{50}$ and convulsions and paralysis as presenting $\mathrm{CS}^{58}$

\section{Summary}

When there is an apparent discrepancy between objective findings and clinical presentation, it is important to consider the possibility of disability due to a psychological mechanism, at the earliest contact. Inaccurate diagnostic labeling may expose patients to unnecessary treatments with the potential for significant side effects. This in turn will have a long term detrimental impact on medical management.

The inter-disciplinary in-patient team management approach in a rehabilitation setting offers the benefits of a comprehensive assessment and treatment for patients with conversion motor paralysis. It is important to note that this diagnosis is temporary and conditional, since there may be a long delay until the appearance of organic findings (as in a systemic disease). A complete medical assessment is essential in order to rule out any possibility of an organic etiology. In as many as $25 \%$ to $50 \%$ of patients diagnosed as conversion, an organic medical diagnosis was found.

\section{References}

1 Parobek VM. Distinguishing conversion disorder from neurologic impairment. J Neurosci Nurs 1997; 29: 128 134.

2 Ford CV, Folks DG. Conversion disorders: an overview. Psychosomatics 1985; 26: $371-383$.

3 Binzer M, Andersen PM, Kullgren G. Clinical characteristics of patients with motor disability due to conversion disorder: a prospective control group study. J Neurol Neurosurg Psychiatry 1997; 63: 83 - 88.

4 American Psychiatric Association: Diagnostic and Statistical Manual of Mental Disorder, Fourth Edition. Washington DC, American Psychiatric Association, 1994.

5 Heruti RJ, Ohry A. The Rehabilitation Team-A Commentary. Am J Phys Med Rehabil 1995; 74: 466468 .

6 Hare E. The history of 'nervous disorders' from 1600 to 1840, and a comparison with modern views. $\mathrm{Br} J$ Psychiatry 1991; 159: $37-45$.

7 Mace CJ. Hysterical conversion. I: A history. $\mathrm{Br} J$ Psychiatry 1992; 161: 369-377.

8 Mai FM, Mersky H. Briquets concept of hysteria: an historical perspective. Can J Psychiatry 1981; 26: 57-63.

9 White MB. Jean-Martin Charcot's contributions to the interface between neurology and psychiatry. Can $J$ Neurol Sci 1997; 24: 254-260.
10 Makari GJ. Dora's hysteria and the maturation of Sigmund Freud's transference theory: a new historical interpretation. J Am Psychoanal Assoc 1997; 45: 1061 1096.

11 Small GW, Propper MW, Randolph ET, Eth S. Mass hysteria among student performers: social relationship as a symptom predictor. Am J Psychiatry 1991; 148: $1200-$ 1205.

12 Lazare A. Current concepts in psychiatry: conversion symptoms. $N$ Engl J Med 1981; 305: 745-748.

13 Miller E. Hysteria: its nature and explanation. $\mathrm{Br} J$ Clin Psychol 1987; 26: $163-173$.

14 Yarom N. A matrix of hysteria. Int J Psychoanal 1997; 78: $1119-1134$.

15 Stefansson JG, Messina JA, Meyerowitz S. Hysterical neurosis, conversion type: clinical and epidemiological considerations. Acta Psychiatr Scand 1976; 53: 119-138.

16 Fishbain DA, Goldberg M, Khalil TM, Asfour SS, Abdel-Moty E, Meagher BR, et al. The utility of electro-myographic biofeedback in the treatment of conversion paralysis. Am J Psychiatry 1988; 145: $1572-$ 1575.

17 Hafeiz HB. Hysterical conversion: a prognostic study. $\mathrm{Br}$ J Psychiatry 1980; 136: 548 - 551.

18 Ziegler FJ. Hysterical conversion reactions. Postgrad Med 1970; 47: 174-178.

19 Chodoff P. The diagnosis of hysteria: an overview. Am J Psychiatry 1974; 131: $1073-1078$.

20 Weintraub MI. Hysterical conversion reactions - a clinical guide to diagnosis and treatment. New York: SP Medical, 1983.

21 Ljundberg L. Hysteria: Clinical, prognostic and genetic study. Acta Psychiatr Scand 1957; 32: 1- 162.

22 Thompson APJ, Sills JA. Diagnosis of functional illness presenting with gait disorder. Arch Phys Med Rehabil 1988; 63: $148-152$.

23 Keane JR. Hysterical gait disorders: 60 cases. Neurology 1989; 39: $586-589$

24 Baker JH, Silver JR. Hysterical paraplegia. J Neurol Neurosurg Psychiatry 1987; 50: 375-382.

25 Cardenas DD, Larson J, Egan KJ. Hysterical paralysis in the upper extremity of chronic pain patients. Arch Phys Med Rehabil 1986; 67: 190-193.

26 Withrington $\mathrm{RH}$, Wynn-Perry CB. Rehabilitation of conversion paralysis. J Bone Joint Surg 1985; 67: 635637.

27 Matas M. Psychogenic voice disorders: literature review and case report. Can J Psychiatry 1991; 36: 363-365.

28 Ophir D, Katz Y, Tavori I, Aladjem M. Functional upper airway obstruction in adolescents. Arch Otolaryngol Head Neck Surg 1990; 116: 1208 - 1209.

29 Weller M, Wiedemann P. Hysterical symptoms in ophthalmology. Doc Ophthalmol 1989; 73: 1-33.

30 Sirota P, Spivac B, Meshulam B. Conversive hallucinations. Br J Psychiatry 1987; 151: $844-846$.

31 Nakaya M. True auditory hallucinations as a conversion symptom. Psychopathology 1995; 28: 214-219.

32 Modai I, Cygielman G, Wijsenbeek H. Hysterical sleep episodes as a manifestation of masked depression. $J$ Nerve Ment Dis 1979; 167: 502-503.

33 Garfield PD, Guze SB. Prognosis and differential diagnosis of conversion reactions. Dis Nerv Syst 1962; 23: $623-631$. 
34 Gould R, Miller BI, Goldberg MA, Benson DF. The validity of hysterical signs and symptoms. J Nerv Ment Dis 1986; 174: 593 - 597.

35 Hudziak JJ, Boffeli TJ, Kreisman JJ, Battaglia MM, Stanger C, Guze SB, Kriesman JJ. Clinical study of the relation of borderline personality disorder to Briquet's syndrome (hysteria), somatization disorder, antisocial personality disorder, and substance abuse disorders [published erratum appears in Am J Psychiatry 1997; 154(1):139]. Am J Psychiatry 1996; 153: 1598 - 1606.

36 Apple Jr DF. Hysterical spinal paralysis. Paraplegia 1989; 7: 428-431.

37 Taylor RL. Distinguishing psychological from organic disorders. New York: Springer, 1990.

38 Johnson EW. Diagnosis of hysterical weakness. Proceedings of the Sixth Annual Scientific Meeting. Australian College of Rehabilitation Medicine. 1986 February $26-$ 28; Sydney.

39 Berry JF, Hillis RE, Hitzman SE. Factitious triplegia: case report. Arch Phys Med Rehabil 1994; 75: 1161 - 1164.

40 Spitz MC. Panic disorder in seizure patients: diagnostic pitfall. Epilepsia 1991; 32 (suppl-1): $33-38$.

41 Beckham JC, Crawford AL, Feldman ME, Kirby AC, Hertzberg MA, Davidson JR, Moore SD. Chronic posttraumatic stress disorder and chronic pain in Vietnam combat veterans. J Psychosom Res 1997; 43(4): $379-389$.

42 Kinzie JD, Goetz RR. A century of controversy surrounding postraumatic stress-spectrum syndromes: the impact on DSM-III and DSM-IV. J Trauma Stress 1996; 9: $159-179$.

43 Stewart TD. Hysterical conversion reactions: some patient characteristics and treatment team reactions. Arch Phys Med Rehabil 1983; 64: 308-310.

44 Vatine JJ, Milun M, Avni J. Inpatient rehabilitation management of conversion disorder with motor dysfunction. J Rehabil Sciences 1996; 9: $122-125$.

45 Delargy MA, Peatfield R, Burt AA. Successful rehabilitation in conversion paralysis. BMJ 1986; 292: $1730-1731$.
46 Keating JJ, Dinan TG, Chua A, Keeling PW. Hysterical paralysis [letter; comment]. Lancet 1990; 336: 15061507.

47 Silver FW. Management of conversion disorder. Am J Phys Med Rehabil 1996; 75: 134-140.

48 Speed J. Behavioral management of conversion disorder: retrospective study. Arch Phys Med Rehabil 1996; 77: $147-154$

49 Solyom C, Solyom L. A treatment program for functional paraplegia/Munchausen syndrome. J Behav Ther Exp Psychiatry 1990; 21: 225-230.

50 Couprie W, Wijdicks EFM, Rooijmans HGM, Van Gijn J. Outcome in conversion disorder: a follow up study. $J$ Neurol Neurosurg and Psychiatry 1995; 58: 750-752.

51 Bird J. The behavioral treatment of hysteria. $\mathrm{Br} J$ Psychiatry 1979; 134: 129-137.

52 White A, Cordin DOC, Coope B. The use of thiopentone in the treatment of non-organic locomotor disorders. $J$ Psychosom Res 1988; 32: 249-253.

53 Mizes JS. The use of contingent reinforcement in the treatment of a conversion disorder: a multiple baseline study. J Behav Ther Exp Psychiatry 1985; 16: 341 - 345.

54 Dickes RA. Brief therapy of conversion reactions: an inhospital technique. Am Psychiatry 1974; 131: 584-586.

55 Khalil TM, Abdel-Moty E, Asfour SS, Fishbain DA, Rosomoff RS, Rosomoff HL. Functional electric stimulation in the reversal of conversion disorder paralysis. Arch Phys Med Rehabil 1988; 69: 545-547.

56 Jellinek DA, Bradford R, Bailey I, Symon L. The role of motor evoked potentials in the management of hysterical paraplegia: case report. Paraplegia 1992; 30: 300-302.

57 Klein MJ, Kewman DG, Sayama M. Behavior modification of abnormal gait and chronic pain secondary to somatization disorder. Arch Phys Med Rehabil 1985; 66: $119-122$

58 Sirota P, Spivac B, Meshulam B. Conversive hallucinations. Br J Psychiatry 1987; 151: 844-846. 